\title{
Ranking of critical species to preserve the functionality of mutualistic networks using the k-core decomposition
}

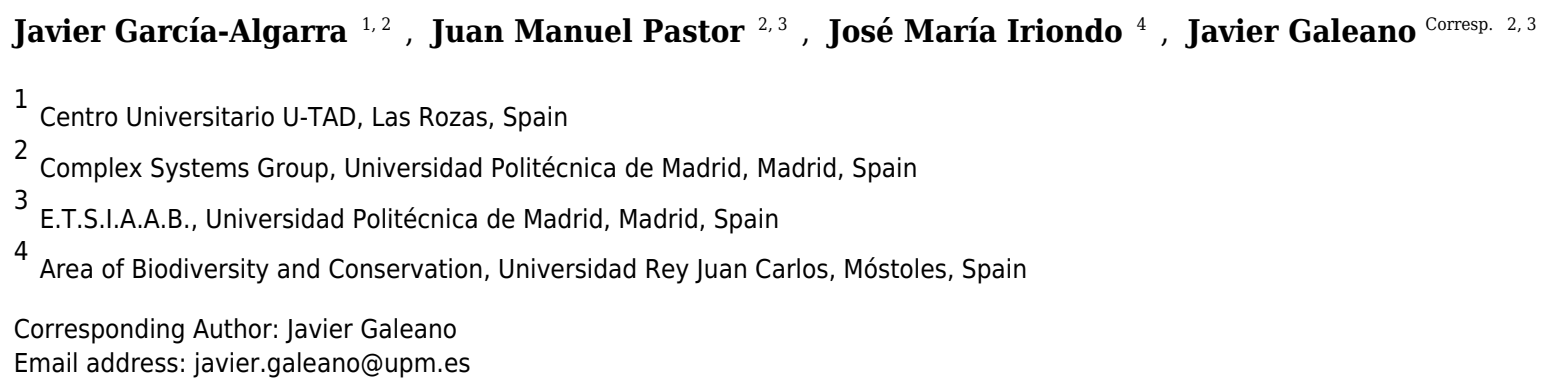

Background. Network analysis has become a relevant approach to analyze cascading species extinctions resulting from perturbations on mutualistic interactions as a result of environmental change. In this context, it is essential to be able to point out key species, whose stability would prevent cascading extinctions, and the consequent loss of ecosystem function. In this study, we aim to explain how the kcore decomposition, sheds light on the understanding the robustness of bipartite mutualistic networks.

Methods. We defined three k-magnitudes based on the k-core decomposition: k-radius, k-degree, and krisk. The first one, k-radius, quantifies the distance from a node to the innermost shell of the partner guild, while k-degree provides a measure of centrality in the k-shell based decomposition. k-risk is a way to measure the vulnerability of a network to the loss of a particular species. Using these magnitudes we analyzed 89 mutualistic networks involving plant pollinators or seed dispersers. Two static extinction procedures were implemented in which k-degree and k-risk were compared against other commonly used ranking indexes.

Results. When extinctions take place in both guilds, k-risk is the best ranking index if the goal is to identify the key species to preserve the giant component. When species are removed only in the primary class and cascading extinctions are measured in the secondary class, the most effective ranking index to identify the key species to preserve the giant component is k-degree. However, MusRank index was more effective when the goal is to identify the key species to preserve the greatest species richness in the second class.

Discussion. The k-core decomposition offers a new topological view of the structure of mutualistic networks. The new k-radius, k-degree and k-risk magnitudes take advantage of its properties and provide new insight into the structure of mutualistic networks. The k-risk and k-degree ranking indexes are especially effective approaches to identify key species to preserve when conservation practitioners focus on the preservation of ecosystem functionality over species richness. 


\title{
Ranking of critical species to preserve the functionality of mutualistic networks using the k-core decomposition
}

\author{
Javier García-Algarra ${ }^{1,4}$, Juan Manuel Pastor ${ }^{1,2}$, José María Iriondo ${ }^{3}$, and \\ Javier Galeano ${ }^{1,2}$ \\ ${ }^{1}$ Complex Systems Group, Universidad Politécnica de Madrid, Madrid, Spain \\ 2E.T.S.I.A.A.B. , Dept. Ingeniería Agroforestal, Universidad Politécnica de Madrid, \\ Madrid, Spain \\ ${ }^{3}$ Area of Biodiversity and Conservation, Universidad Rey Juan Carlos, Móstoles, Spain \\ ${ }^{4}$ Centro Universitario U-TAD, Las Rozas, Spain \\ Corresponding author: \\ Javier Galeano ${ }^{1}$ \\ Email address: javier.galeano@upm.es
}

\section{ABSTRACT}

Background. Network analysis has become a relevant approach to analyze cascading species extinctions resulting from perturbations on mutualistic interactions as a result of environmental change. In this context, it is essential to be able to point out key species, whose stability would prevent cascading extinctions, and the consequent loss of ecosystem function. In this study, we aim to explain how the k-core decomposition, sheds light on the understanding the robustness of bipartite mutualistic networks. Methods. We defined three k-magnitudes based on the k-core decomposition: k-radius, k-degree, and $\mathrm{k}$-risk. The first one, $\mathrm{k}$-radius, quantifies the distance from a node to the innermost shell of the partner guild, while k-degree provides a measure of centrality in the k-shell based decomposition. $\mathrm{k}$-risk is a way to measure the vulnerability of a network to the loss of a particular species. Using these magnitudes we analyzed 89 mutualistic networks involving plant pollinators or seed dispersers. Two static extinction procedures were implemented in which k-degree and k-risk were compared against other commonly used ranking indexes.

Results. When extinctions take place in both guilds, k-risk is the best ranking index if the goal is to identify the key species to preserve the giant component. When species are removed only in the primary class and cascading extinctions are measured in the secondary class, the most effective ranking index to identify the key species to preserve the giant component is k-degree. However, MusRank index was more effective when the goal is to identify the key species to preserve the greatest species richness in the second class.

Discussion. The k-core decomposition offers a new topological view of the structure of mutualistic networks. The new $\mathrm{k}$-radius, k-degree and k-risk magnitudes take advantage of its properties and provide new insight into the structure of mutualistic networks. The k-risk and k-degree ranking indexes are especially effective approaches to identify key species to preserve when conservation practitioners focus on the preservation of ecosystem functionality over species richness.

\section{INTRODUCTION}

Biotic interaction networks play an essential role in the stability of ecosystems (Tylianakis et al., 2010), as well as in the maintenance of biodiversity (Bascompte et al., 2006). Because community dynamics greatly depend on the way species interact, these networks have been described as the "biodiversity architecture" (Bascompte and Jordano, 2007). Network analysis has become an important approach to provide information on community organization and to predict dynamics and species extinctions in response to ecosystem disturbance (Tylianakis et al., 2010; Thébault and Fontaine, 2010; Traveset and Richardson, 2014). Among other assessments, these studies can point out key species, whose stability would prevent cascading extinctions, and the consequent loss of biodiversity (Sole and Montoya, 
2001; Suweis et al., 2013; Dakos et al., 2014; Santamaría et al., 2015). Research on cascading species extinctions as a result of perturbations in biotic interactions has tackled two main issues: the different ways to rank a hypothetical extinction sequence and the robustness and fragility measures (Pocock et al., 2012; Domínguez-García and Muñoz, 2015). There are different strategies both to sort species according to their importance and to measure their influence on extinction. For instance, in early studies on the resilience of food webs Dunne et al. ranked species by degree (i.e., the number of interactions) using three different scenarios of removal: a) from the species with the highest degree to the species with the lowest degree; b) from the lowest to the highest; c) species selected in a random way (Dunne et al., 2002). Memmott et al. worked the same idea to assess the robustness of mutualistic communities, removing active species (in this context, pollinators or seed dispersers) and measuring the fraction of remaining passive species (plants) (Memmott et al., 2004).

An observed property of mutualistic interactions is the existence of generalists, highly interconnected, and specialists, with few interactions linked to the generalists, but rarely among them. The nucleus of interactions among generalists seems to be the foundation of resilience. This property has been traditionally identified with nestedness (Bascompte et al., 2003), although not all mutualistic communities are nested (Joppa et al., 2010; Staniczenko et al., 2013). There are new approaches to describe this structure in a more general way as a core-periphery organization (Csermely et al., 2013; Rombach et al., 2014). The core is the set of central and densely interconnected nodes. Ties of periphery are sparse and usually with nodes of the core.

Identification of key species for community preservation is another active field of research. Besides classical measures of node centrality such as closeness, degree or betweenness (Callaway et al., 2000), new rankings based on the well-known Google's PageRank ${ }^{\mathrm{TM}}$ algorithm are now available for ecological networks (Allesina and Pascual, 2009). There are efficient ways to find out these key species in bipartite networks that have been tested in one of the static extinction scenarios we use in this paper (Tacchella et al., 2012; Domínguez-García and Muñoz, 2015).

Another scenario to identify the key species relative to the role species play in the architecture of the network is the study of modularity (Guimerà and Amaral, 2005; Blondel et al., 2008; Guimerà and Sales-Pardo, 2009). A module is a group of species more closely connected to each other than to species in other modules. This ideas have been used in the study of mutualistic networks, estimating how much modular mutualistic network are (Olesen et al., 2007). This analysis allows classifying the nodes into different roles but does not provide a species ranking for possible extinction scenarios. Furthermore, we focus on the relationship of the species with the innermost core of the network, because this core is the cornerstone to understand the functionality of the network.

In this paper, we aim to explain how the $k$-core decomposition, sheds light on the understanding of robustness in mutualistic networks. The tool classifies the nodes of the network in shells, as in an onionlike structure with the most connected nodes in its center. Taking into account these basic topological properties, the decomposition helps to assess in detail the structure of mutualism and enlightens on the processes of species extinction cascades. Derived from the k-core decomposition we introduce three new magnitudes, hereafter called $k$-magnitudes, that describe network compactness, defined as the connection to the innermost shell of the network, (k-radius), combined quantity and quality of interactions (k-degree) and species vulnerability to trigger extinction cascades (k-risk). We assess the best criteria for identifying the species for which the networks are most vulnerable to cascade extinctions by comparing k-degree and k-risk ranking criteria with ranking by well-known indexes and applying them in two network destruction procedures. To conduct the test, we use one of the most complete available data sets (Fortuna et al., 2014).

\section{MATERIALS AND METHODS}

\section{Data}

We have analyzed the Web of life collection (Fortuna et al., 2014), comprised by 89 mutualistic networks, with 59 communities of plants and pollinators and 30 of seed dispersers (http: / / www . web-of-life. es/). There are 57 communities with binary adjacency matrix (i.e., the interaction between the two species is recorded but not its strength), and 32 with weighted matrix, where the strength is accounted for. Network sizes range from 6 to 997 species, the minimum number of links is 6 and the maximum is 2933 . 


\section{Decomposition and k-magnitudes}

The idea of core decomposition was first described by Seidman to measure local density and cohesion in social graphs (Seidman, 1983). It has been successfully applied to visualize large systems and networks (Alvarez-Hamelin et al., 2005; Kitsak et al., 2010; Zhang et al., 2010; Barberá et al., 2015).

The $k$-core of a network is a maximal connected sub-network of degree greater or equal than $k$. That means that each node in the sub-network is tied to at least $k$ other nodes in the same sub-network.

A simple algorithm to perform the $k$-core decomposition prunes links of nodes of degree equal or less than $k$ (Batagelj and Zaversnik, 2003). The process starts removing links with one of their edges in a node of degree 1. This procedure is recursive and ends when all the remaining nodes have at least two links. The isolated nodes are the 1-shell. Then it continues with $k=2$, and so on. After performing the $k$-decomposition, each species belongs to one of the $k$-shells (Fig. 1). The $m$-core includes all nodes of $m$-shell, $m+1$-shell... with $m$ ranging from 1 to the max $k$ index of that particular network. For instance, the 2-core of Fig. 1 is the union of the 1-shell and the 2-shell.

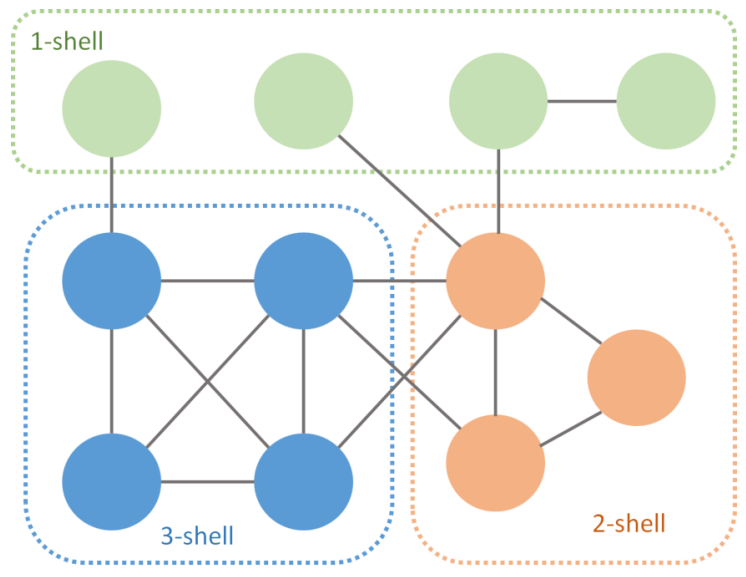

Figure 1. $k$-core decomposition of a fictional network. Green links are pruned during the first iteration, orange during the second and blue during the last one.

Mutualistic networks are bipartite, with two guilds of species (plant-pollinator or plant-seed disperser in the studied collection). Links among nodes of the same class are forbidden. We will call these guilds $A$ and $B$.

Based on the k-core decomposition, we define three k-magnitudes. In order to quantify the distance from a node to the innermost shell of the partner guild, we define $k_{\text {radius }}$. The $k_{\text {radius }}$ of node $m$ of guild $A$ is the average distance to all species of the innermost shell of guild $B$. We call this set $N^{B}$.

$$
k_{\text {radius }}^{A}(m)=\frac{1}{\left|N^{B}\right|} \sum_{j \in N^{B}} \text { dist }_{m j} \quad m \in A, \forall j \in B
$$

where dist $_{m j}$ is the shortest path from species $m$ to each of the $j$ species that belong to $N^{B}$. The minimum possible $k_{\text {radius }}$ value is 1 for one node of the innermost shell directly linked to each one of the innermost shell set of the opposite guild.

To obtain a measure of centrality in this $k$-shell based decomposition, we define $k_{\text {degree }}$ as

$$
k_{\text {degree }}^{A}(m)=\sum_{j} \frac{a_{m j}}{k_{\text {radius }}^{B}(j)} \quad m \in A, \forall j \in B
$$

where $a_{m j}$ is the element of the interaction matrix that represents the link, considered as binary. If the network is weighted, $a_{m j}$ will count as 1 for this purpose if there is interaction, 0 otherwise. It could be understood that $k_{\text {degree }}(m)$ is a fine-grained measure, whereas degree would be a coarse-grained measure. $k_{\text {degree }}(m)$ is like a "continuous" degree where each node $i$ linked to node $m$ adds the inverse of its $k_{\text {radius }}(j)$. Generalists score high $k_{\text {degree }}$, whereas specialists, which have only one or two links, with 
similar $k_{\text {radius }}$, score lower $k_{\text {degree }}$. This magnitude reminds the definition of the Harary index (Plavšić et al., 1993) but only considering paths from the nodes tied from $m$ to the nodes of the innermost shell.

Figure 2 shows how $k_{\text {degree }}$ works for one particular network. There are many nodes with the same degree value (Fig. 2A), such as specialists with just one or two links, that from a ranking point of view are equivalent. On the contrary $k_{\text {degree }}$, maps the degree distribution onto a more continuous one (Fig. 2B), because of the contribution of the inverse of $k_{\text {radius }}$. In Fig. $2 \mathrm{C}$ the cumulative distributions of both indexes are overimposed over the degree scale (We perform the linear regression $k_{\text {degree }}=\alpha \times$ degree and fit the $k_{\text {degree }}$ distribution to the degree values).

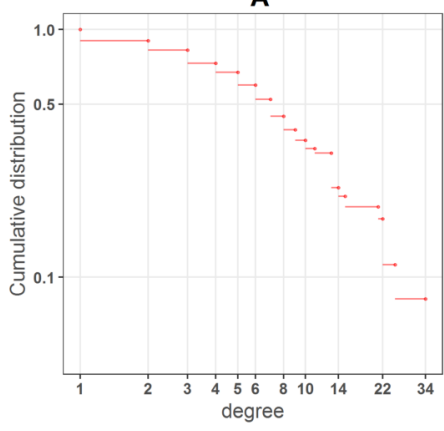

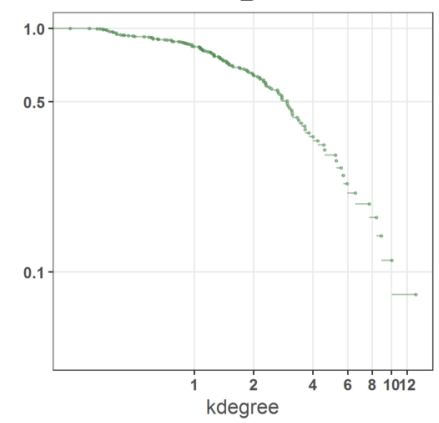

C

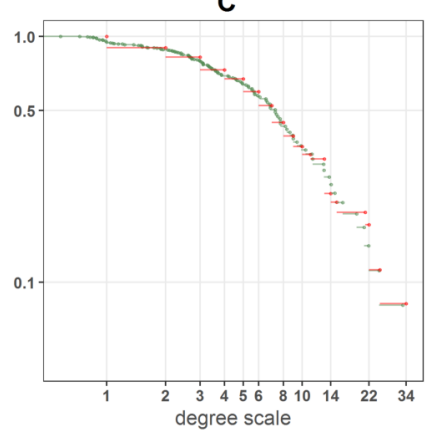

Figure 2. (A) Degree, (B) $k_{\text {degree }}$ and (C) overimposed $k_{\text {degree }}$ distributions of a big plant pollinator community in Central Los Andes, Chile Arroyo et al. (1982).

Finally, we introduce $k_{r i s k}$ as a way to measure how vulnerable is a network to the loss of a particular species:

$$
k_{\text {risk }}^{A}(m)=\sum_{j} a_{m j}\left(k_{\text {shell }}^{A}(m)-k_{\text {shell }}^{B}(j)\right)+\varepsilon k_{\text {shell }}^{A}(m) \quad m \in A, \forall j \in B, k_{\text {shell }}^{B}(j)<k_{\text {shell }}^{A}(m)
$$

The $k_{\text {risk }}$ of a given species is the sum of the k-shell differences to all the nodes of the other guild on a lower $k$-shells to which it is connected. Each one is weighted by the difference of the $k$ indexes. The second element of equation 3 is meant to solve ties among species when they belong to different $k$-shells, and is a very small quantity (in our implementation we use 0.01 , two orders of magnitude lesser than the sum).

In an intuitive way, if we remove one node strongly connected to others of lower $k$-shells, these species are in high risk of being dragged by the primary extinction. On the other hand, the extinction is much less dangerous for the species of higher $k$-shells linked to the same node, because they enjoy more redundant paths towards the network nucleus.

\section{Applying the $k$-magnitudes to a network}

Fig. 3 is an small seed disperser network with five species of plants, four species of thrushes and eleven links. We call, by convention, guild $A$ the set of plants, and guild $B$ the set of birds. The $k$-core decomposition was performed with the $\mathrm{R}$ igraph package (Csardi and Nepusz, 2006). The maximum $k$ index is 2 . The four bird species belong to 2-shell; there are three plant species in 1-shell and two in 2-shell. In this example each species of 2-shell is directly tied to all species of the opposite guild 2-shell, but this is not a general rule.

The shortest path from plant species 2 to each of the four bird species of 2-shell is 1, because of the direct links. So, $k_{\text {radius }}^{A}(2)$ is 1 . The same reasoning is valid for plant species 1 . The reader may check that the $k_{\text {radius }}$ of bird species of 2-shell is 1 as well, measuring their shortest paths to plants species 1 and 2.

Computation of this magnitude is simple although a bit more laborious for 1 -shell plant species. We work plant species 4 as an example. First, we find the shortest paths to each bird species of 2-shell. Shortest paths are depicted with different colors. Plant species 4 is tied to seed disperser species 1 , so distance is 1 . On the other hand, there is no direct link with bird species 2. Shortest path is pl4-disp1$p l 2$-disp2, and distance is 3 . It is easy to check that distances from plant species 4 to bird species 3 and 4 


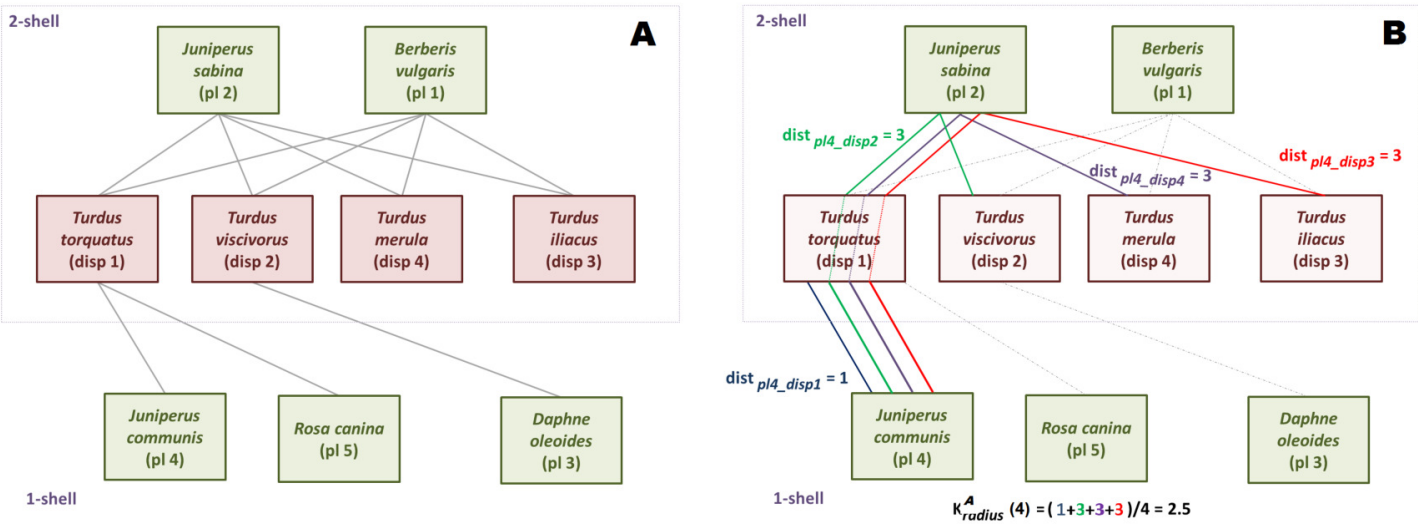

Figure 3. Computation of the $\boldsymbol{k}$-magnitudes. Seed disperser network in Santa Bárbara, Sierra de Baza (Spain) (Jordano, 1993). A: Decomposed network. B: Computing $k_{\text {radius }}^{A}(4)$.

are also 3. Once we have found the four distances, we compute $k_{\text {radius }}^{B}(4)$ as the average of $1,3,3$ and 3 , that is 2.5 .

The values of $k_{\text {degree }}$ are straightforward to compute. For instance, the $k_{\text {degree }}$ of disperser species 1 is:

$$
k_{\text {degree }}^{B}(1)=\frac{1}{k_{\text {radius }}^{A}(1)}+\frac{1}{k_{\text {radius }}^{A}(2)}+\frac{1}{k_{\text {radius }}^{A}(4)}+\frac{1}{k_{\text {radius }}^{A}(5)}=2.8
$$

The last $k$-magnitude we defined was $k_{\text {risk }}$. We use again the disperser species 1 as example. Links to species of the same or upper $k$-shells are irrelevant to compute $k_{\text {risk }}$, so only plant species 4 and 5 are taken into account.

$$
k_{\text {risk }}^{B}(1)=k_{\text {shell }}^{B}(1)-k_{\text {shell }}^{A}(4)+k_{\text {shell }}^{B}(1)-k_{\text {shell }}^{A}(5)+\varepsilon k_{\text {shell }}^{B}(1)=(2-1)+(2-1)+0.01 \times 2=2.02
$$

This magnitude may seem counter-intuitive, because the $k_{\text {risk }}$ of a highly connected species like plant 1 is 0.02 , almost the same of that of peripheral plant $3(0.01)$. This is because plant 1 has no ties with lower $k$-shell animal species. The $k_{\text {risk }}$ ranks species to assess resilience, it has not an absolute meaning. It just tells us that it is more dangerous for the network to remove the disperser 1 than plant 1 , and plant 1 than plant 3 (Table 1).

The k-magnitudes of the example network are shown in table 1.

\begin{tabular}{|r|r|r|r|r|}
\hline Species & $k_{\text {shell }}$ & $k_{\text {radius }}$ & $k_{\text {degree }}$ & Rank by $k_{\text {risk }}$ \\
\hline $\mathrm{p} 11$ & 2 & 1 & 4 & $3^{\text {rd }}$ \\
$\mathrm{p} 12$ & 2 & 1 & 4 & $3^{\text {rd }}$ \\
$\mathrm{p} 13$ & 1 & 2.5 & 1 & $4^{\text {th }}$ \\
$\mathrm{p} 14$ & 1 & 2.5 & 1 & $4^{\text {th }}$ \\
$\mathrm{p} 15$ & 1 & 2.5 & 1 & $4^{\text {th }}$ \\
disp1 & 2 & 1 & 2.8 & $1^{\text {st }}$ \\
disp2 & 2 & 1 & 2.4 & $2^{\text {nd }}$ \\
disp3 & 2 & 1 & 2 & $3^{\text {rd }}$ \\
disp4 & 2 & 1 & 2 & $3^{\text {rd }}$ \\
\hline
\end{tabular}

Table 1. K-magnitudes of the network of Fig. 3.

\section{Extinction procedures}

In order to rank the critical species to preserve the functionality of mutualistic networks and visualize eventual decomposition of the giant component (i.e., the highest connected component of a given network), we carried out two static extinction procedures. Static assumption implies that there is not rewiring (e.g., 
plants that have lost their pollinators are not pollinated by other insects), despite this kind of network reorganization is observed in nature (Ramos-Jiliberto et al., 2012; Goldstein and Zych, 2016; Timóteo et al., 2016). Nodes are ranked once, before the procedure starts, as in most of robustness assessments studies (Memmott et al., 2004; Kaiser-Bunbury et al., 2010; Domínguez-García and Muñoz, 2015).

In the first method, one species is removed each step, in decreasing order according to the chosen index, no matter to which guild it belongs. Four ranking indexes are compared: $k_{r i s k}, k_{\text {degree }}$, degree and eigenvector centrality. The $k$ indexes were computed with the R package kcorebip; degree and eigenvector centrality with the degree and evcent functions of the igraph package.

To estimate the damage caused to the network, the fraction of remaining giant component was used. The procedure stops when this ratio is equal or less than 0.5 . To break ties, we ran 100 experiments for each network and index, shuffling species with the same ranking value. The percentage of removed species needed to get to 0.5 of the remaining giant component is used to measure the performance of the ranking. The lower the percentage of removed species, the more efficient the ranking is in destroying the network. The top performer scores the least average removal percentage. (Fig. 4).

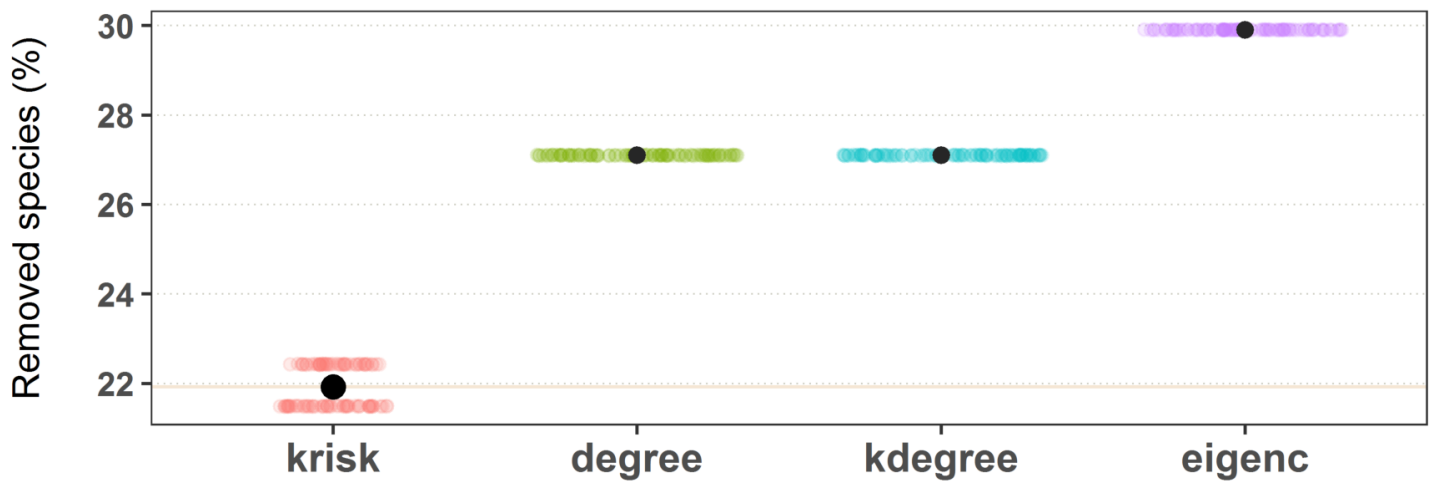

Figure 4. Results of the first extinction procedure for the pollinator network number 10 of the web of life collection. Performance of the four ranking indexes for a pollinator community described by Elberling and Olesen in Zackenberg Station (Greenland). Individual dots are the results of each experiment while black dots are the average values. The horizontal dispersion is just added jitter for visualization. Performance plots of the 89 networks are available at the github repository, more details in the additional information subsection.

The second extinction procedure that we followed is more common in the literature. Only animal species are actively removed (primary extinctions); secondary extinctions happen when nodes become isolated (Memmott et al., 2004).

The fraction of surviving plant species is measured as a function of the removed fraction of animal species (Fig. 5A,C) and the area under the curve is the value to compare performance. We averaged the results of 100 repetitions.

In this case, in addition to the four indexes of the first experiment, we include MusRank a non-linear ranking algorithm for bipartite networks (Tacchella et al., 2012), inspired by PageRank (Allesina and Pascual, 2009). This algorithm is not valid for the first extinction method. Domínguez-García and Muñoz showed that MusRank achieves excellent performance for this extinction procedure (Domínguez-García and Muñoz, 2015).

In the second extinction procedure, we also measured the fraction of remaining giant component (Fig. 5B,D) . Extinction sequences are identical, the only difference is that both magnitudes are measured for each step. 

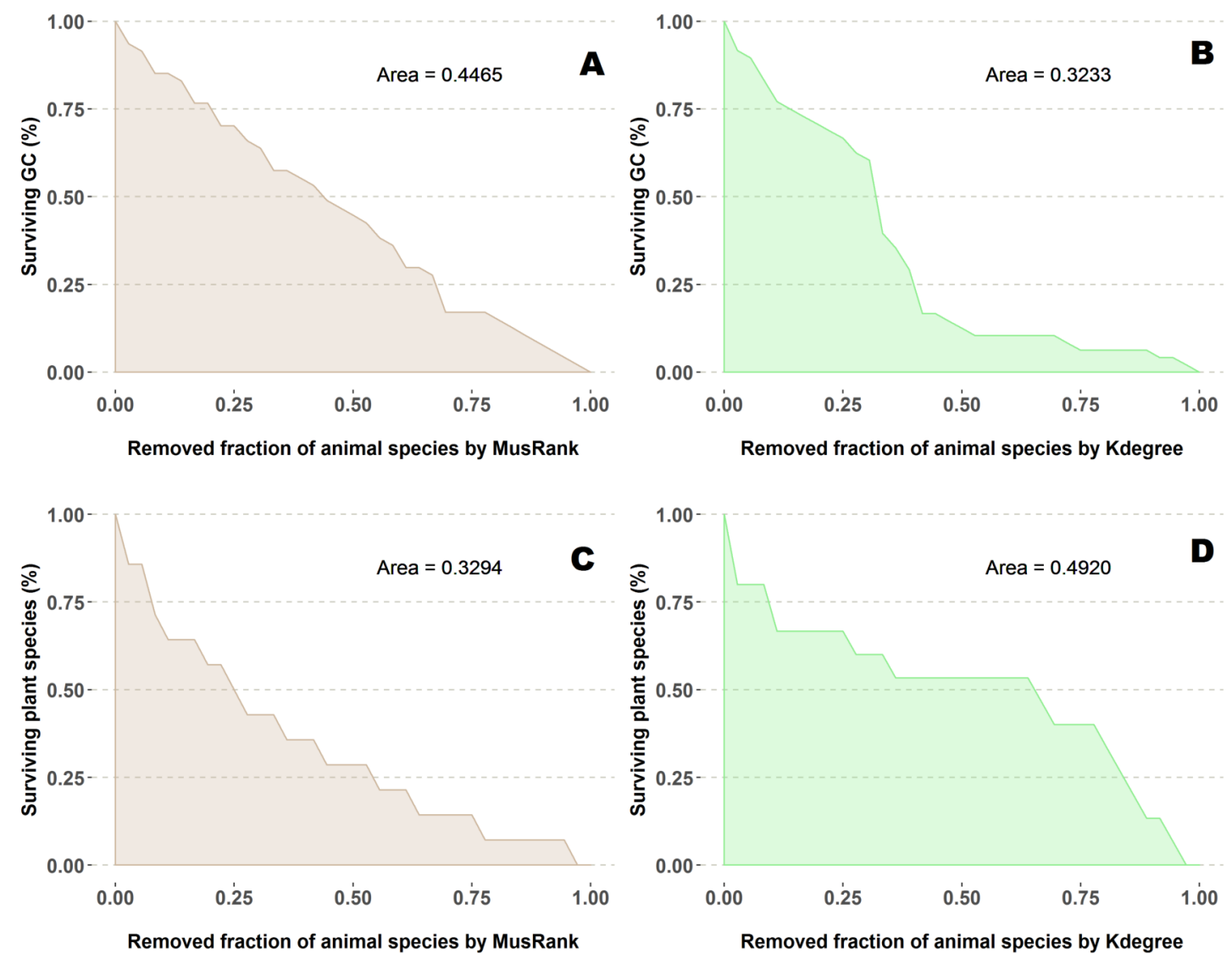

Figure 5. Extinction curves of the second algorithm for the pollination network network number 07 of the collection, Suffolk, UK (Dicks et al., 2002). A,C: Percentage of surviving giant component (GC) and percentage of surviving plant species removing animal species ranked by MusRank. B,D: Percentage of surviving giant component (GC) and percentage of surviving plant species removing animal species ranked by $k_{\text {degree }}$. AUCs of the 89 networks are available at the github repository, more details in the additional information subsection. 


\section{RESULTS}

\section{First extinction method}

$k_{\text {risk }}$ was the ranking method with the lowest average species removal percentage to destroy half of the Giant Component in most of the networks (67 out of 89 networks) (see supplemental material, Table S1). Figure 6 shows the performance comparison of the four ranking criteria. There are some ties, more frequent when networks are small. Network size is the key factor to explain why the performance range is so wide.

As size increases, the removal percentage to break the giant component decreases (Fig. 7). When the network is big, the primary extinction of key nodes triggers an important amount of secondary ones. If the community has 100 or more species, $k_{\text {risk }}$ is even a better predictor of the most damaging extinction sequence and outperforms the other indexes for 28 out of 32 networks.

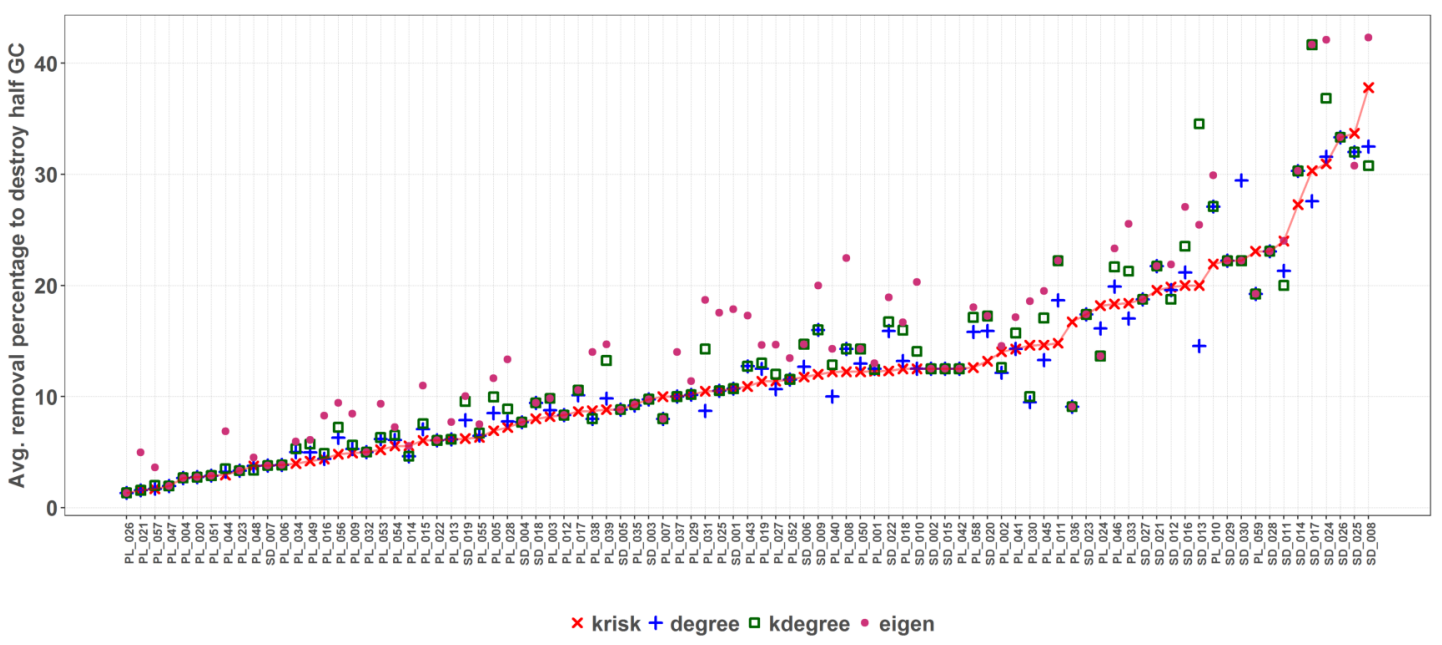

Figure 6. First extinction method results. The average percentage of removed species to destroy the Giant Component (GC) is depicted for each network and ranking index. Under the $X$ axis, the name of each network as coded in the web of life database. The overall top performer is $k_{r i s k}$ (see supplemental material, Table S1). Species are ordered by the percentage of primary extinctions, ranked by $k_{\text {risk }}$. The red line joins the $k_{r i s k}$ destruction percentage values as a visual reference to compare them with those of the other indexes. 


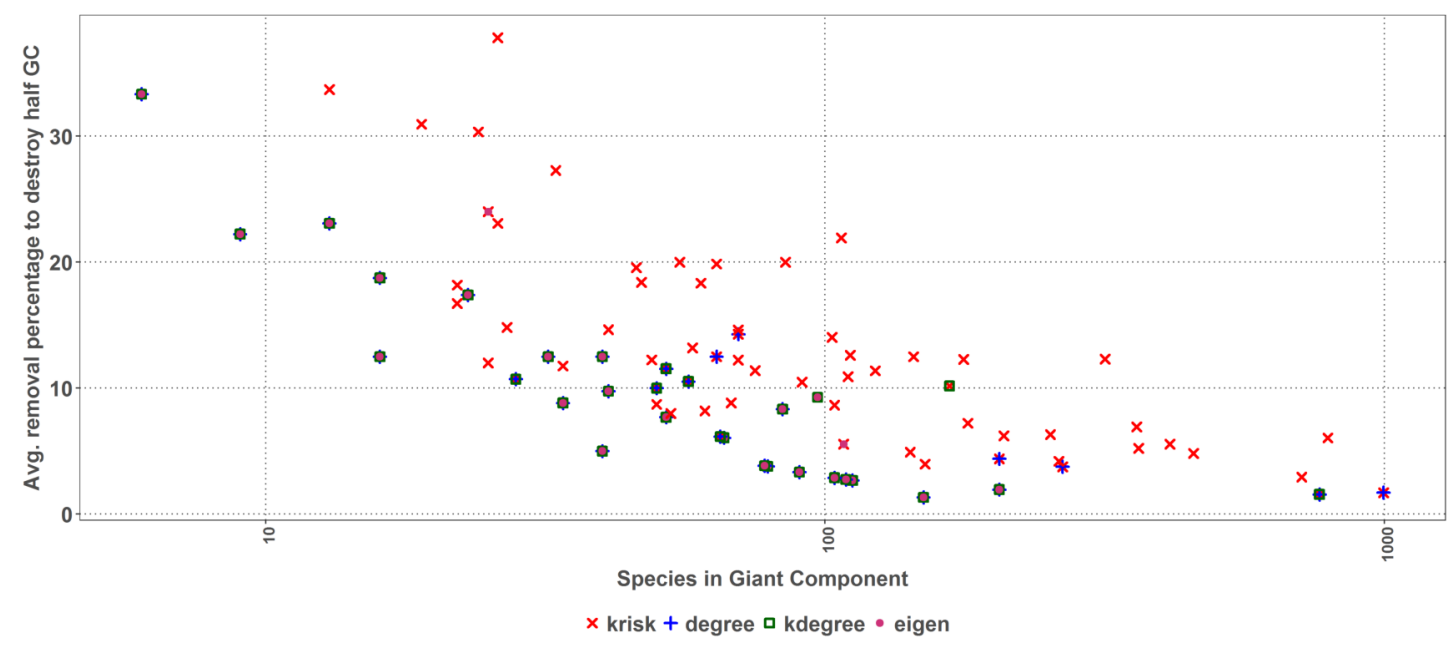

Figure 7. First extinction procedure. Average percentage of removed species to destroy the Giant Component of the top performer ranking as a function of the network size. Dots represent the best result for each network, when there are ties among several rankings for the same network, they overlap 


\section{Second extinction method}

MusRank ranking method had the lowest area under the extinction curve for 85 of the 89 studied networks (Figure 8), and in the other 4 the difference is so small that may be just an effect of the averaging procedure. So, MusRank is the optimal ranking index to destroy the network following this algorithm.

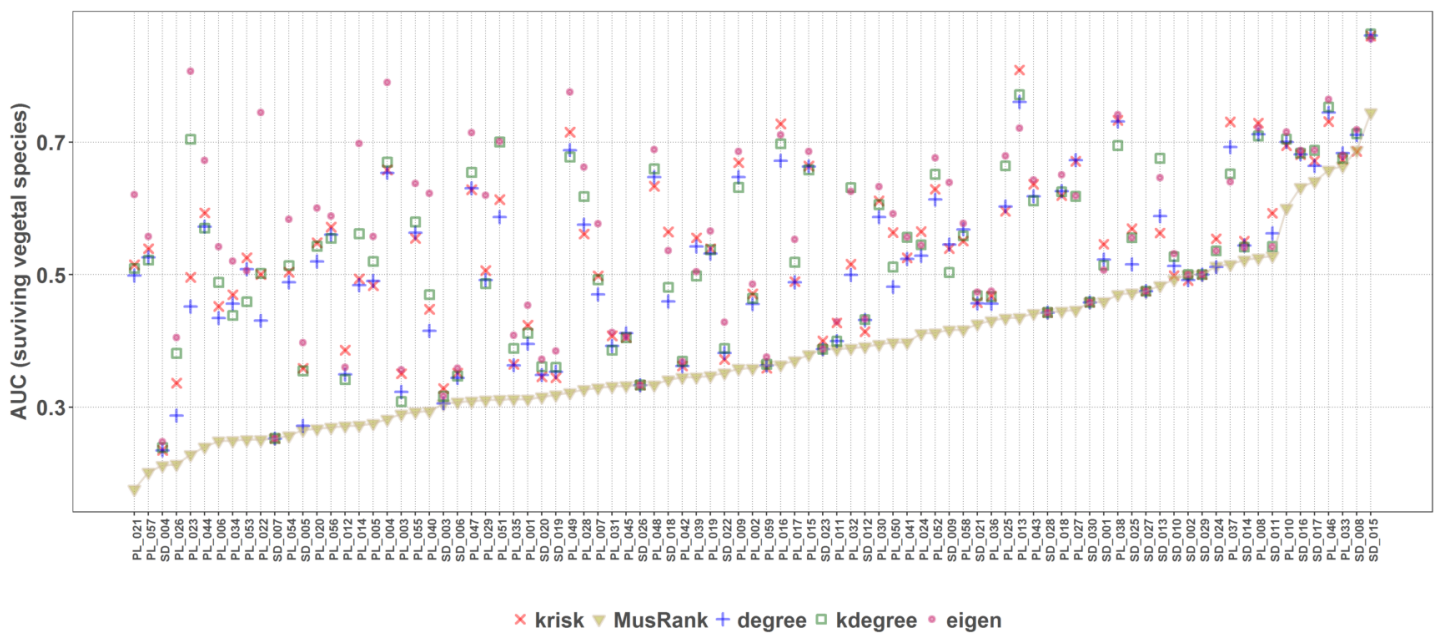

Figure 8. Second extinction procedure, Area Under the Curve of the surviving fraction of plant species as a function of the fraction of removed animal species. AUCs are plotted for each network and ranking. The overall top performer is MusRank (see supplementary material, Table S2). The solid line joins the MusRank values. Species are ordered by the percentage of primary extinctions, ranked by MusRank.

On the contrary, when the efficiency of the network destruction was measured through the area under the curve of the surviving Giant Component fraction the MusRank index had the highest values, placing it as the least efficient ranking method according to this criterion (Figure 9). In this case, $k_{\text {degree }}$ is the most efficient index for 42 out of 89 networks. We must underline that the extinction sequences are the same, the only difference is the measured output.

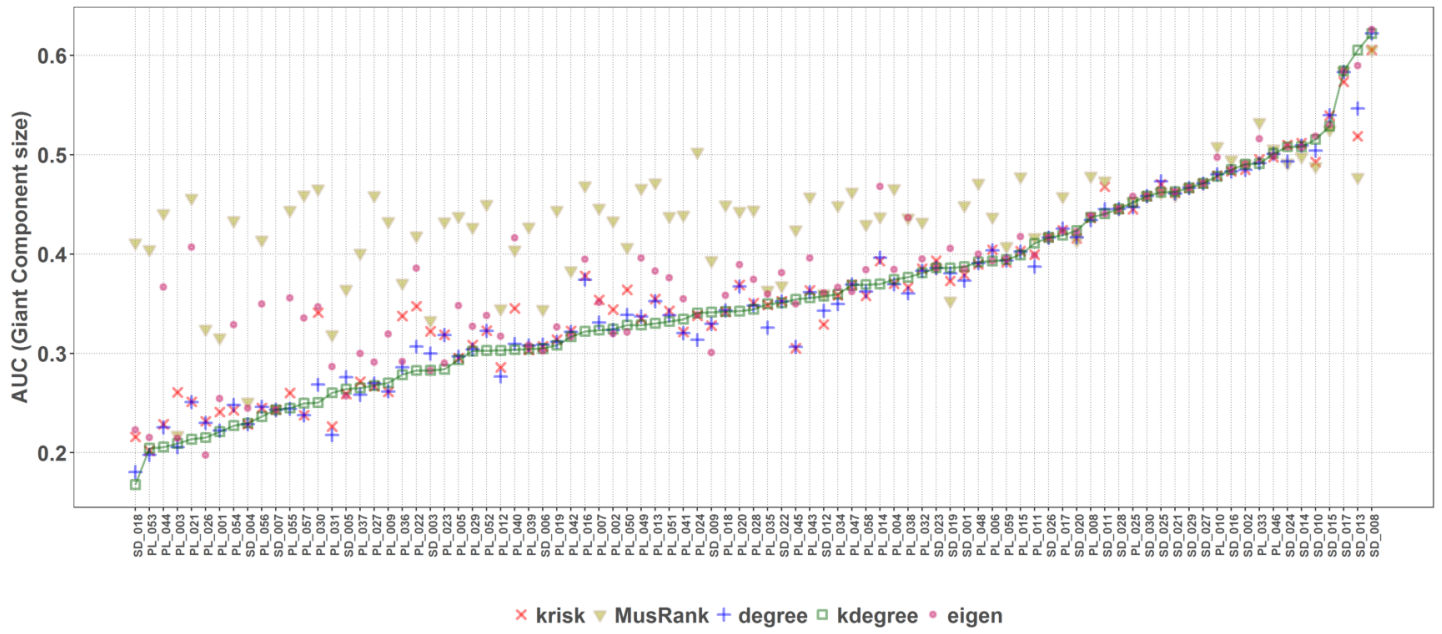

Figure 9. Second extinction procedure, Area Under the Curve of the surviving fraction of the original size of the Giant Component as a function of the fraction of removed animal species. AUCs are plotted for each network and ranking. The overall top performer is $k_{\text {degree }}$ (see supplementary material, Table S3). The solid line joins the $k_{\text {degree }}$ values. Species are ordered by the percentage of primary extinctions, ranked by $k_{\text {degree }}$.

We have worked out one example (Fig. 5) to explain this shocking difference in performance depending on the measured outcome. On the upper row (subplots $A$ and $B$ ), the difference for both ranking indexes 
when measuring the giant component. While this magnitude decreases at a constant pace for MusRank, there is a sharp reduction of the component size when one third of animal species are removed following the $k_{\text {degree }}$ ranking. On the lower row (subplots $C$ and $D$ ), opposite results are obtained when accounting for the fraction of surviving plant species.

The destruction of this pollinator network sheds light on the root cause of the difference. The network has 36 pollinator and 16 plant species (Fig.10A), 2 of them are outside the giant component. When the 13 top animal species ranked by MusRank are removed (pollinators 3, 1, 7, 15, 32, 6, 14,33, 13,31, 8, 16, 10), the community reaches the degraded structure of Fig.10B. The size of the giant component is $27(54 \%$ of the original), and there are 23 pollinator and 6 plant species.
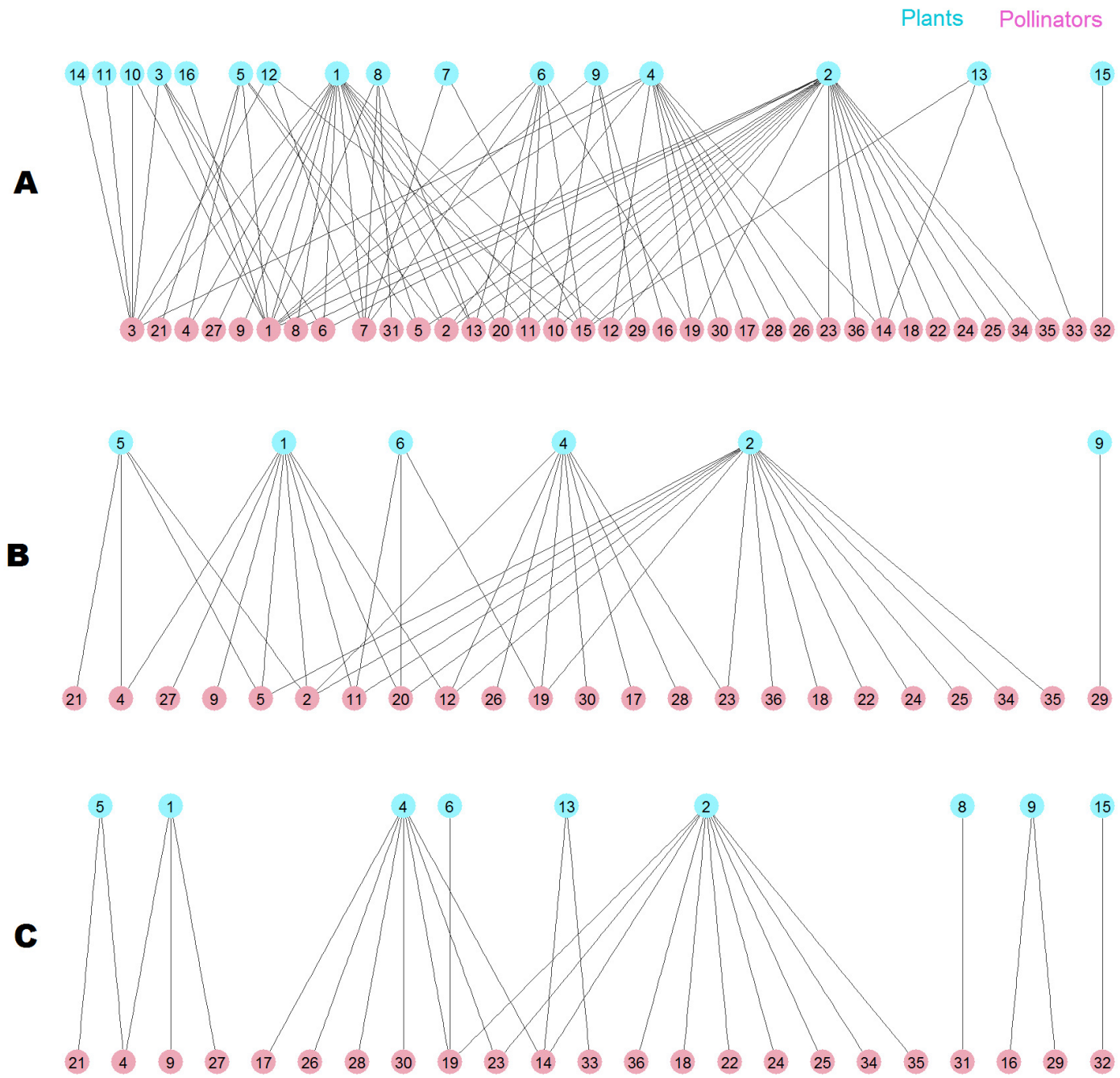

Figure 10. Pollinator network 007 (Dicks et al., 2002). A: Original configuration; B: Structure after the removal of the 13 top MusRank-ranked animal species. C: Structure after the removal of the 13 top $k_{\text {degree }}$-ranked animal species

If we remove the 13 top animal species ranked by $k_{\text {degree }}$ (pollinators $1,3,7,13,15,2,11,20,12,8,6,5,10$ ) instead, the community structure is that of Fig.10C. Now, the size of the giant component is 19 (38\% of the original), and there are 23 pollinator and 9 plant species. MusRank has killed more plant species, but the giant component is clearly smaller ranking by $k_{\text {degree }}$.

\section{DISCUSSION}

The $k$-core decomposition offers a new topological view of the structure of mutualistic networks. We have defined three new magnitudes to take advantage of their properties. Network compactness is described 
by $k_{\text {radius }}$, a measure of average proximity to top generalists of the partner guild. Second, $k_{\text {degree }}$ maps each node's degree onto a finer grain distribution. It has not only information on the number of neighbors but also on how they are connected to the innermost shell. Finally, $k_{\text {risk }}$ is set to identify species whose disappearance poses a greater risk to the entire network.

Comparing the $k$-magnitudes based extinction indexes $\left(k_{\text {degree }}\right.$ and $\left.k_{\text {risk }}\right)$ with those routinely used when extinctions take place in both guilds, $k_{r i s k}$ is the best rank if the goal is to identify the key species to preserve most of the giant component. $k_{\text {risk }}$ identifies species linked to a high number of nodes of lower $k$-shells. These species provide vulnerability to the network because their extinction may drag many of the species with lower $k$-shells they are linked to, to extinction as well, as they do not enjoy redundant paths to the innermost shell.

Applying the well-known method of removing species of the primary class and measuring the extinctions in secondary class, the most effective extinction sequence, if the goal is to identify the key species to preserve most of the giant component, is $k_{\text {degree }}$. However, if the goal is to identify the key species to preserve the greatest species richness in the second class (e.g., plants in a plant-pollinator mutualistic network), the best criterion is MusRank as Fig. 8 makes clear. These results confirm those obtained by Domínguez-García and Muñoz (2015), over a larger network collection (89 in this work vs. 67 in the original paper).

The most striking result of the second method is how different performance is for a same ranking index, depending on the magnitude we measure. The root cause lies on the definitions of the indexes themselves. MusRank is optimal to destroy the plant guild. It identifies the most important active nodes of the bipartite network because of how they are linked to the most vulnerable passive ones. It was designed to excel with this extinction sequence and works with local properties. On the other hand, $k_{\text {degree }}$ is an excellent performer to destroy the giant component. It contains information on how nodes are connected to the innermost shell, and ranks higher those nodes strongly tied to that stable nucleus. This new approach to network robustness could be also applied to other types of networks in which the integrity of the giant component is more important than the number of remaining nodes, for example in communications or epidemic spread networks.

In summary, in this study, we show that the new $k$-core decomposition derived indexes, $k_{\text {risk }}$ and $k_{\text {degree }}$ provide a new insight into the structure of mutualistic networks. This insight is particularly useful because these indexes fair much better than other traditionally used ranking indexes, when the aim is to identify the species that are key to preserving the interactions and the functionality of the community. As complex network studies on mutualistic interactions are already being used to suggest conservation policies, it is of utmost importance to have a clear framework of what the conservation practitioners look for when implementing conservation and restoration plans. The static view of considering biodiversity conservation as the mere conservation of a list of species has long been substituted by a new paradigm which looks at conservation from a dynamic viewpoint in which species interactions and the functionality of the ecosystems play a major role (Heywood and Iriondo, 2003).

\section{ADDITIONAL INFORMATION AND DECLARATIONS}

\section{Code}

The $\mathrm{R}$ code for $k$-core decomposition and plotting has been published as a package at https: / / zenodo . org/badge/latestdoi/58714207.

The rest of software is available at https://zenodo.org/badge/latestdoi/78195863 Reproducibility instructions are detailed in the README. md file

\section{Extinction plots}

All extinction plots are available at https://zenodo.org/badge/latestdoi/78195863 Subdirectory graphs /FIRST contains the performance plots of the first algorithm for each network. Subdirectory graphs / AREAS contains the normalized AUC plots of the second algorithm for each network. Under the subdirectory graphs/python there are two entries GCdestroymethod and PLdestroymethod with the performance comparison plots of the second algorithm when measuring surviving percentage of the Giant Component or surviving percentage of PLant species. 


\section{REFERENCES}

Allesina, S. and Pascual, M. (2009). Googling food webs: can an eigenvector measure species' importance for coextinctions? PLoS Comput Biol, 5(9):e1000494.

Alvarez-Hamelin, J. I., Dall'Asta, L., Barrat, A., and Vespignani, A. (2005). k-core decomposition: A tool for the visualization of large scale networks. arXiv preprint cs/0504107.

Arroyo, M. T. K., Primack, R., and Armesto, J. (1982). Community studies in pollination ecology in the high temperate andes of central chile. i. pollination mechanisms and altitudinal variation. American journal of botany, pages 82-97.

Barberá, P., Wang, N., Bonneau, R., Jost, J. T., Nagler, J., Tucker, J., and González-Bailón, S. (2015). The critical periphery in the growth of social protests. PloS One, 10(11).

Bascompte, J. and Jordano, P. (2007). Plant-animal mutualistic networks: the architecture of biodiversity. Annual Review of Ecology, Evolution, and Systematics, pages 567-593.

Bascompte, J., Jordano, P., Melián, C. J., and Olesen, J. M. (2003). The nested assembly of plant-animal mutualistic networks. Proceedings of the National Academy of Sciences, 100(16):9383-9387.

Bascompte, J., Jordano, P., and Olesen, J. M. (2006). Asymmetric coevolutionary networks facilitate biodiversity maintenance. Science, 312:431-433.

Batagelj, V. and Zaversnik, M. (2003). An o (m) algorithm for cores decomposition of networks. arXiv preprint cs/0310049.

Blondel, V. D., Guillaume, J.-L., Lambiotte, R., and Lefebvre, E. (2008). Fast unfolding of communities in large networks. Journal of statistical mechanics: theory and experiment, 2008(10):P10008.

Callaway, D. S., Newman, M. E., Strogatz, S. H., and Watts, D. J. (2000). Network robustness and fragility: Percolation on random graphs. Physical review letters, 85(25):5468.

Csardi, G. and Nepusz, T. (2006). The igraph software package for complex network research. InterJournal, 1695(5):1-9.

Csermely, P., London, A., Wu, L.-Y., and Uzzi, B. (2013). Structure and dynamics of core/periphery networks. Journal of Complex Networks, 1(2):93-123.

Dakos, V., Carpenter, S. R., van Nes, E. H., and Scheffer, M. (2014). Resilience indicators: prospects and limitations for early warnings of regime shifts. Philosophical Transactions of the Royal Society of London B: Biological Sciences, 370(1659):1-10.

Dicks, L., Corbet, S., and Pywell, R. (2002). Compartmentalization in plant-insect flower visitor webs. Journal of Animal Ecology, 71(1):32-43.

Domínguez-García, V. and Muñoz, M. A. (2015). Ranking species in mutualistic networks. Scientific Reports, 5.

Dunne, J. A., Williams, R. J., and Martinez, N. D. (2002). Network structure and biodiversity loss in food webs: robustness increases with connectance. Ecology letters, 5(4):558-567.

Fortuna, M. A., Ortega, R., and Bascompte, J. (2014). The web of life. arXiv preprint abs/1403.2575.

Goldstein, J. and Zych, M. (2016). What if we lose a hub? experimental testing of pollination network resilience to removal of keystone floral resources. Arthropod-Plant Interactions, 10(3):263-271.

Guimerà, R. and Amaral, L. A. N. (2005). Functional cartography of complex metabolic networks. Nature, 433(7028):895-900.

Guimerà, R. and Sales-Pardo, M. (2009). Missing and spurious interactions and the reconstruction of complex networks. Proceedings of the National Academy of Sciences, 106(52):22073-22078.

Heywood, V. H. and Iriondo, J. M. (2003). Plant conservation: old problems, new perspectives. Biological conservation, 113(3):321-335.

Joppa, L. N., Montoya, J. M., Solé, R., Sanderson, J., and Pimm, S. L. (2010). On nestedness in ecological networks. Evolutionary ecology research. 2010; 12: 35-46.

Jordano, P. (1993). Geographical ecology and variation of plant-seed disperser interactions: southern Spanish junipers and frugivorous thrushes. Springer.

Kaiser-Bunbury, C. N., Muff, S., Memmott, J., Müller, C. B., and Caflisch, A. (2010). The robustness of pollination networks to the loss of species and interactions: a quantitative approach incorporating pollinator behaviour. Ecology Letters, 13(4):442-452.

Kitsak, M., Gallos, L. K., Havlin, S., Liljeros, F., Muchnik, L., Stanley, H. E., and Makse, H. A. (2010). Identification of influential spreaders in complex networks. Nature Physics, 6(11):888-893.

Memmott, J., Waser, N. M., and Price, M. V. (2004). Tolerance of pollination networks to species extinctions. Proceedings of the Royal Society of London B: Biological Sciences, 271(1557):2605-2611. 
Olesen, J. M., Bascompte, J., Dupont, Y. L., and Jordano, P. (2007). The modularity of pollination networks. Proceedings of the National Academy of Sciences, 104(50):19891-19896.

Plavšić, D., Nikolić, S., Trinajstić, N., and Mihalić, Z. (1993). On the harary index for the characterization of chemical graphs. Journal of Mathematical Chemistry, 12(1):235-50.

Pocock, M. J. O., Evans, D. M., and Memmott, J. (2012). The robustness and restoration of a network of ecological networks. Science, 335(6071):973-977.

Ramos-Jiliberto, R., Valdovinos, F. S., Moisset de Espanés, P., and Flores, J. D. (2012). Topological plasticity increases robustness of mutualistic networks. Journal of Animal Ecology, 81(4):896-904.

Rombach, M. P., Porter, M. A., Fowler, J. H., and Mucha, P. J. (2014). Core-periphery structure in networks. SIAM Journal on Applied mathematics, 74(1):167-190.

Santamaría, S., Galeano, J., Pastor, J. M., and Méndez, M. (2015). Removing interactions, rather than species, casts doubt on the high robustness of pollination networks. Oikos.

Seidman, S. B. (1983). Network structure and minimum degree. Social Networks, 5(3):269-287.

Sole, R. and Montoya, J. M. (2001). Complexity and fragility in ecological networks. Proc. R. Soc. Lond. $B, 268: 2039-2045$.

Staniczenko, P. P., Kopp, J. C., and Allesina, S. (2013). The ghost of nestedness in ecological networks. Nature Communications, 4:1391.

Suweis, S., Simini, F., Banavar, J. R., and Maritan, A. (2013). Emergence of structural and dynamical properties of ecological mutualistic networks. Nature, 500(7463):449-452.

Tacchella, A., Cristelli, M., Caldarelli, G., Gabrielli, A., and Pietronero, L. (2012). A new metrics for countries' fitness and products' complexity. Scientific Reports, 2.

Thébault, E. and Fontaine, C. (2010). Stability of ecological communities and the architecture of mutualistic and trophic networks. Science, 329(5993):853-856.

Timóteo, S., Ramos, J. A., Vaughan, I. P., and Memmott, J. (2016). High resilience of seed dispersal webs highlighted by the experimental removal of the dominant disperser. Current Biology, 26(7):910-915.

Traveset, A. and Richardson, D. M. (2014). Mutualistic interactions and biological invasions. Annual Review of Ecology, Evolution, and Systematics, 45:89-113.

Tylianakis, J., Laliberte, E., Nielsen, A., and Bascompte, J. (2010). Conservation of species interaction networks. Biological Conservation, 143:2270-2279.

Zhang, H., Zhao, H., Cai, W., Liu, J., and Zhou, W. (2010). Using the k-core decomposition to analyze the static structure of large-scale software systems. The Journal of Supercomputing, 53(2):352-369. 